\title{
A Summary of Cloud Computing and Its Technology Research
}

\author{
Xin Sui ${ }^{1, a}$ and Yi Sui ${ }^{2, b *}$ \\ ${ }^{1}$ College of Humanities and Sciences of Northeast Normal University, Changchun, \\ 130117 ,P.R.China \\ ${ }^{2}$ China Telecom, Beijing Branch Beijing,100124 ,P.R.China \\ a Suixin1997@126.com; b ericsuiyi@qq.com \\ *The Corresponding author
}

Keywords: Virtual Technology; Cloud Computing; IT; Internet

\begin{abstract}
In the context of the 1980s grid computing and the virtual technology of twenty-first Century, cloud computing is recognized by the industry as a new type of delivery model and resource. Many research institutions have seen the value of cloud computing. Cloud computing is a major change in IT technology after the rapid development of personal computers and the rapid development of the Internet. This article mainly introduces the related knowledge and related technologies of cloud computing.
\end{abstract}

\section{Cloud Computing}

Cloud computing is a new generation of information technology that integrates grid computing, cluster computing, virtualization, distributed processing and distributed computing. There are many definitions of cloud computing. MIT defines the cloud computing as a new IT service mode that can get the infrastructure, application platform and software through the Internet self service and on demand payment.

California Berkeley defines cloud computing as: terminal users' software and hardware are delivered by cloud providers in the form of services in the network.

IBM believes that cloud computing is a shared service mode that delivers information through the network, in which users of the cloud service only need to interact with the service, without the need to care about how the underlying infrastructure is implemented.

\section{Service Mode of Cloud Computing}

The service mode of cloud computing, that is, cloud services, and the cloud service provides personalized products, is a business service model to meet the different needs of various users on the market.

The delivery mode of cloud computing includes IaaS, Infrastructure as a Service, PaaS, Platform as a Service, SaaS, Service, etc.

Infrastructure as a Service

IaaS is a cloud computing model that encapsulates the cloud infrastructure, that is, hardware resources such as storage, input and output devices, memory, servers and so on, so that they can be provided to users.

IaaS vendors generally have some of the following functions:

Through real-time monitoring of resources, we can ensure efficient and orderly operation of infrastructure.

Automate all resources from creation to deployment, and then to the process used.

Provide effective security management to ensure legitimate access to and use of infrastructure and resources. 
In IaaS, every requirement can be satisfied by increasing the available resources. If users do not use them, they can release these resources, that is, fast elastic computing. Users only need to have low cost of hardware, storage and computing power, thus reducing the cost of hardware.

PaaS means that a service provider virtualized the software application platform, managed and allocated it as a pool of resources, and provided it to the user as a service.

The platform application service is an extension of the definition of cloud computing, configuring the operating system and platform, and providing the load capability of the service platform for direct application. In this service mode, users do not consider the coordination problem between computers, and the platform is responsible for dynamic expansion of resources.

Platform application services generally provide the following functions:

An integrated development environment is provided to enable users to develop and test applications.

Provide various forms of services to the upper level.For application level management and monitoring, we calculate accurately the resources consumed by the application and then charge.

Platform application services effectively solve the problems of expensive platform, complex platform management and unscientific user needs.

SaaS means that a service provider encapsulates a specific software into a service, deployed on a server, and users access the various applications provided by the cloud service provider through terminal devices.

To achieve this, suppliers should have the following functions:

The supplier can visit anywhere at any time, and users can access SaaS services if they can access the Internet.

Support for open protocols to provide more convenient use.

Provide security mechanism, implement security mechanism on the user side to protect them, and protect the security of cloud data.

SaaS services reduce the high cost and high availability cloud platform services of the platform. SaaS services have assembled a complete set of services to be delivered to users in full agreement.

In the SaaS service mode, users do not have to spend too much on hardware, software, and managers as they used to. They only need a terminal device connected to the Internet, so they can use all kinds of software at anytime and anywhere.

\section{The core Technology of Cloud Computing Platform}

Cloud computing is a technology evolution from traditional technology to new technology and a highly integrated computing mode of information data and structural processing capability.

Cloud computing is the storage and transmission of data through the network, and there are many core technologies in data management, storage and programming mode. It mainly includes virtualization technology, resource management and scheduling, distributed file system, cloud computing platform management and so on.

\section{Virtualization Technology}

Virtualization technology is one of the most important core technologies of cloud computing, and is an important technical cornerstone of cloud computing. In the field of computer science, virtualization technology is not only confined to the concept of virtual machine, but also represents the abstraction of computing resources.

In the definition of virtualization technology, the following comparisons are easy to understand. Virtualization technology is a method to allocate computing resources (hardware and software resources). It breaks the strict division between data center, storage, server, network, data and application physical equipment, and separates the hardware, software, network and other different levels. To achieve the purpose of dynamic use of force resources, centralized management of virtual resources, improve the flexibility and flexibility of the system structure, improve service, reduce cost and other purposes. 
Virtualization technology mainly refers to the application of virtualization technology, which can expand the capacity of hardware and simplify the process of configuring system software effectively. By reconfiguring, it can adapt to more operating systems and greatly reduce the cost of virtual machines.

Virtualization technology can help isolate hardware and software levels, and can divide individual resources into several virtualized resources.

\section{Advantages of Virtualization Technology}

It effectively reduces the operation cost of the enterprise. The IT cost of an enterprise includes purchase cost and maintenance cost. The cost of purchase mainly includes the purchase of hardware, software, services and licenses. The cost of operation and maintenance mainly includes the cost of infrastructure management and maintenance. Virtualization provides a simpler and more powerful management interface, making administrators out of a large number of management tasks that deal with problems such as servers and compatibility.

Virtualization technology provides the function of dynamic migration. Dynamic migration can move virtual machines from one physical server to another without interrupting service. The virtual machine can move freely in the resource pool, and the administrator can mobilize the resources of the virtual machine in time.

By using virtualization technology, multiple servers can be integrated into a physical server to improve the utilization rate of physical servers. The virtual integration of the server greatly improves the server's memory utilization, storage rate, and the utilization of CPU, and the utilization rate of the whole network is also greatly improved.

Virtualization technology improves the capability of continuous and reliable operation. Users can easily and quickly use the virtual machine snapshot to be divided into virtual machine image files. When the hardware system fails, or when the hardware fails, the virtual machine is dynamically migrated to a backup, which greatly improves the availability of the service.

Using virtual machine technology, deploying an application is to deploy a packaged operating system and application virtual machine. The process of deploying a virtual machine is less time-saving than the traditional deployment. It is not easy to make mistakes, no manual intervention is needed, and the cost of deployment is reduced.

In server virtualization, the hardware is abstracted first, resource allocation, management, and the realization of the isolation of different virtual machines, and then the software is virtualized. Virtualization technology in cloud computing can standardize all kinds of resources such as storage, computing and software, and integrate resources. We should rationally divide resources units and make full use of hardware resources. A dynamic apology is performed on different physical nodes to achieve load independent performance independent of application. The merging of multiple virtual machine computing nodes to the same physical node saves electricity and closes idle physical nodes.

\section{Acknowledgements}

2018 Jilin Province Education Department "Thirteen-Five" Science and Technology Project "Enterprise Private Cloud Construction Design and Research ", Project No.: JJKH20181306KJ.

\section{Summary}

Cloud computing is a new computing mode, and cloud computing resources are huge. Many servers are distributed in different locations. The management platform of the cloud computing system can make a large number of servers work together and realize the reliable operation of large-scale systems through intelligence. 


\section{References}

[1] Wei Sulin. Cloud Computing Deployment Mode and Its Key Technology Research [J]. Fujian Computer, 2017,33 (11): 7-8.

[2] Xu Xinxin. Analysis of Cloud Computing and Its Key Technologies [J]. Information and Computer, 2013 (05): 121-122.

[3] Hu Ying. Cloud Computing and Its key Technology Research [J]. Software Guide, 2016,15 (08): 159-161.

[4] Chen Cong. Cloud Computing Security and Security Architecture and Its Key Technologies [J]. Shandong Industrial Technology, 2016 (16): 289-290.

[5] Shen Xu. Cloud Computing and Tts Key technology analysis [J]. Technology Innovation and Application, 2017 (16): 104.

[6] Liu Guo. Cloud Computing and Its Key Technologies. [J]. Information Security and Technology, 2015,6 (09): 41-43.

[7] Zhao Yan. Cloud Computing and Its Key technology Research [J]. Computer Disc Software and Applications, 2014,17 (12): 45-46. 